Preprints are preliminary reports that have not undergone peer review.

They should not be considered conclusive, used to inform clinical practice, or referenced by the media as validated information.

\title{
Ablation rate after radioactive iodine therapy in patients with differentiated thyroid cancer at intermediate or high risk of recurrence: a systematic review and a meta- analysis
}

\section{Michele Klain}

University of Naples Federico II Department of Advanced Biomedical Sciences: Universita degli Studi di Napoli Federico II Dipartimento di Scienze Biomediche Avanzate

\section{Carmela Nappi}

University of Naples Federico II Department of Advanced Biomedical Sciences: Universita degli Studi di Napoli Federico II Dipartimento di Scienze Biomediche Avanzate

\section{Emilia Zampella}

University of Naples Federico II Department of Advanced Biomedical Sciences: Universita degli Studi di Napoli Federico II Dipartimento di Scienze Biomediche Avanzate

\section{Valeria Cantoni}

University of Naples Federico II Department of Advanced Biomedical Sciences: Universita degli Studi di Napoli Federico II Dipartimento di Scienze Biomediche Avanzate

\section{Roberta Green}

University of Naples Federico II Department of Advanced Biomedical Sciences: Universita degli Studi di Napoli Federico II Dipartimento di Scienze Biomediche Avanzate

\section{Leandra Piscopo}

University of Naples Federico II Department of Advanced Biomedical Sciences: Universita degli Studi di Napoli Federico II Dipartimento di Scienze Biomediche Avanzate

\section{Fabio Volpe}

University of Naples Federico II Department of Advanced Biomedical Sciences: Universita degli Studi di Napoli Federico II Dipartimento di Scienze Biomediche Avanzate

\section{Mariarosaria Manganelli}

University of Naples Federico II Department of Advanced Biomedical Sciences: Universita degli Studi di Napoli Federico II Dipartimento di Scienze Biomediche Avanzate

\section{Elisa Caiazzo}

University of Naples Federico II Department of Advanced Biomedical Sciences: Universita degli Studi di Napoli Federico II Dipartimento di Scienze Biomediche Avanzate

\section{Martin Schlumberger}

University of Naples Federico II Department of Advanced Biomedical Sciences: Universita degli Studi di Napoli Federico II Dipartimento di Scienze Biomediche Avanzate

\section{Alberto Cuocolo ( $D$ cuocolo@unina.it )}

University of Naples Federico II Department of Advanced Biomedical Sciences: Universita degli Studi di Napoli Federico II Dipartimento di Scienze Biomediche Avanzate https://orcid.org/0000-0003-3431-7658 


\section{Research Article}

Keywords: Differentiated thyroid cancer, 131I, Meta-analysis, Intermediate-risk, High-risk

Posted Date: March 19th, 2021

DOI: https://doi.org/10.21203/rs.3.rs-324463/v1

License: (c) (i) This work is licensed under a Creative Commons Attribution 4.0 International License. Read Full License

Version of Record: A version of this preprint was published at European Journal of Nuclear Medicine and Molecular Imaging on June 18th, 2021. See the published version at https://doi.org/10.1007/s00259-021-05440-x. 


\section{Abstract \\ Purpose}

We performed a systematic review and a meta-analysis to investigate the successful ablation rate after radioiodine (RAI) administration in patients with differentiated thyroid cancer (DTC) at intermediate-high risk of recurrence.

\section{Methods}

A comprehensive literature search of the PubMed, Scopus and Web of Science databases was conducted according to the PRISMA statement.

\section{Results}

The final analysis included 9 studies accounting for 3103 patients at intermediate-high risk of recurrence. In these patients, the successful ablation rates ranged from $51-94 \%$ with a $71 \%$ pooled successful ablation and was higher in intermediate $(72 \%)$ than in high $(52 \%)$ risk patients. Despite the rigorous inclusion standards, a significant heterogeneity among the evaluated studies was observed. Higher administered RAl activities are associated with a lower successful ablation rate in the whole population and in the subgroup of high-risk patients. Further, pooled recurrence rate in intermediate risk patients achieving successful ablation was only $2 \%$ during the subsequent 6.4 year follow-up while the pooled recurrence rate was $14 \%$ in patients who did not achieve a successful ablation.

\section{Conclusion}

In a large sample of 3103 patients at intermediate-high risk of persistent/recurrent disease, $71 \%$ of patients achieved a successful ablation. In these intermediate-risk patients, the probability of subsequent recurrence is low and most recurrence occurred in those with already abnormal findings at the first control.

\section{Introduction}

After total thyroidectomy with or without lymph node dissection for differentiated thyroid cancer (DTC), radioiodine (RAI) may be administered for 3 main goals [1]: remnant ablation (to facilitate the detection of recurrent disease by destroying post-operative remnants of non-tumoral thyroid tissue and to permit initial staging with a whole-body scan), adjuvant therapy (to decrease the risk of recurrence by destroying suspected, but unproven persistent disease), or therapy (to treat known persistent disease). Successful ablation (SA) can be assessed some months later by an undetectable serum thyroglobulin (Tg) level on L-T4 using a sensitive method and unremarkable findings at neck ultrasound [2-4].

The indication for post-operative RAI administration takes into account the American Thyroid Association (ATA) threetiered risk system [1] that classifies patients as low, intermediate or high-risk of recurrence. Whereas post-surgical RAI therapy is usually not indicated in low-risk patients, it is generally recommended in intermediate and high-risk patients. Intermediate-risk group includes patients with microscopic extra-thyroid extension, aggressive histology, vascular invasion, $>5$ lymph node metastases (N1) with all $\mathrm{N} 1<3 \mathrm{~cm}$, multifocal papillary microcarcinoma with ETE or BRAFV600E mutation and RAl-avid metastatic foci in the neck on the first whole-body RAl scan. High-risk patients show macroscopic ETE, incomplete tumor resection, biochemical or structural evidence of distant metastatic disease, 
any $\mathrm{N} 1>3 \mathrm{~cm}$ or follicular thyroid cancer with extensive vascular invasion. Although literature offers a huge armamentarium of data on survival benefit of RAI in intermediate-high risk patients, to our knowledge a metaanalysis on SA rate in these patients $[5,6]$ has not yet been performed.

Therefore, we performed a systematic review and a meta-analysis to investigate the SA rate after RAI administration in patients with DTC at intermediate-high risk of recurrence.

\section{Methods}

\section{Search strategy}

This meta-analysis followed the Preferred Reporting Items for Systematic Reviews and Meta- Analyses (PRISMA) statement (see Supplementary material for PRISMA Checklist) [7], and registered as 242409 in the PROSPERO database (University of York, UK; http://www.crd.york.ac.uk/PROSPERO/). An English literature search was performed using the PubMed and Embase databases to identify articles published from 2010 until June 2020. This search was restricted to data obtained in adults and was conducted using the following key words: "differentiated thyroid cancer" OR "DTC", "thyroid neoplasm", "prognosis", "outcome”, "follow-up", "radioactive iodine therapy" OR "RAI therapy", "I131 ablation", "thyroglobulin" OR "Tg".

\section{Study selection}

The title and abstract of potentially relevant studies were screened for appropriateness before retrieval of the full article by two reviewers (L.P. and F.V.), and disagreements were resolved by consensus.

The selected full-published reports were retrieved and the same reviewers independently performed a second-step selection based on the eligibility criteria; disagreements were resolved by consensus.

In addition, the bibliographies of retrieved articles were manually reviewed for potential additional citations.

\section{Study eligibility and data extraction}

Each study was initially identified considering journal, authors, and year of publication. To harmonize the predictors of interest, a study was considered eligible if all of the following criteria were met: 1) Data were available on age, gender and administered RAI activity, histopathology and extent of surgery. 2) the study presented data of adult subjects with differentiated thyroid cancer at intermediate or high risk of recurrence after RAl therapy; we excluded studies on low-risk patients only and we excluded the low-risk patients in studies that included both low and intermediate/high-risk patients [1]. Investigations that considered different risk classification, such as the AJCC/TNM staging system [8] were included in the final analysis only if a detailed description of clinical and histopathological characteristics of patients was provided, allowing patient categorization with the ATA risk classification basis (e.g. studies only including patients having microscopic extra-thyroidal extension which are considered intermediate ATA risk or studies clearly providing data on patients with pT3-pT4 tumor or metastasis who were considered ATA high risk); 3) the study included at least 100 subjects; 4) follow-up after RAI therapy for at least 1 year; 5) the study provided data on SA after RAl therapy defined as absence of abnormal findings at neck ultrasonography and undetectable serum $\mathrm{Tg}$ in the absence of anti-Tg antibodies [2,3]. In case of multiple studies reported from the same research group, potential cohort duplication was avoided by including only the study on the largest number of patients.

\section{Assessment of the methodological quality of studies}


All studies were assessed for methodological quality using Joanna Briggs Institute (JBI) Prevalence Critical Appraisal Tool [9]. The criteria address the following issues: Ensuring a representative sample, ensuring appropriate recruitment, ensuring an adequate sample size, ensuring appropriate description and reporting of study subjects and setting, ensuring data coverage of the identified sample is adequate, ensuring the condition was measured reliably and objectively, ensuring appropriate statistical analysis, ensuring confounding factors/subgroups/differences are identified and accounted for. These questions can be answered with four possible responses: yes, no, unclear or not applicable [9]. Two reviewers (V.C. and R.G.) evaluated the risk of bias in each eligible study. and disagreements between reviewers were resolved by consensus.

The two reviewers completed the screening process independently.

Disagreement in the process of answering questions was discussed until consensus was reached. A final decision of yes (favorable scenario, "+"), no (unfavorable scenario, "-"), or unclear (mixed scenario, "+/-") was made by the reviewers after systematic discussion. If the answers to all the signal problems were "yes", a low risk of bias was attributed to the study; if the answers to all the signal problems had one or more "no" or "unclear" values, an unclear risk of bias was used; if the answers to all the signal problems contained at least one "no" but no "yes" answers, a high risk of bias was attributed.

\section{Statistical analysis}

We used a systematic analytic approach to compute the pooled SA rate after RAI therapy in patients with DTC from all eligible studies. Metaprop was used to perform meta-analysis of proportions close to or at the margins, $0 \%$ or $100 \%$. Metaprop pools proportions and presents a weighted subgroup and overall pooled estimates with inversevariance weights obtained from a random-effect model [11]. Heterogeneity of the included studies was examined by using the I-squared $\left(\mathrm{I}^{2}\right)$ statistic, to reflect the percentage of total variation across studies [11], assigning adjectives of low, moderate, and high to $\mathrm{I}^{2}$ values of $25 \%, 50 \%$, and $75 \%$. According to the Cochrane handbook, $\mathrm{I}^{2}>50 \%$ reflects a substantial heterogeneity [12]. Therefore, a random effect model is used to combine data in the meta-analysis [13]. The possibility of publication bias in the present study was examined by using Egger's test [14]. Publication bias was graphically examined by the funnel plot and also formally assessed with the regression test of asymmetry described by Egger et al [14]. When statistical heterogeneity was substantial, meta-regression analysis was performed to assess if study-level variables such as age, gender and RAI administered activity were associated with pooled SA rate. When it was feasible, we evaluated the pooled SA rate separately in DTC patients at intermediate and high risk of recurrence. We also evaluated the pooled recurrent disease rate in DTC patients who achieved SA when data were available. All analyses were performed using Stata, version 15.1 (StataCorp, College Station, TX). Two-sided $P$ values $<0.05$ were considered statistically significant.

\section{Results}

\section{Study selection}

The complete literature search is presented in Fig. 1. The initial search identified 819 potentially eligible citations. Among these, 119 were identified as duplicates and thus removed, leaving 700 records. The reviewers, after the evaluation of their titles and abstracts of these studies removed 655 citations. Then, each investigator blindly reviewed the full text of the remaining 45 articles, and 36 articles were excluded. The remaining 9 articles included a total of 6675 patients, 3103 of whom at intermediate-high risk of recurrence and were the basis of the present metaanalysis. 


\section{Characteristics of the included studies}

Demographic data and clinical characteristics of the 3103 patients included in the meta-analysis are detailed in Table 1 [15-23]. Study sample size ranged from 152 to 627 subjects. Mean age was $47 \pm 1$ years, with the proportion of women ranging from $66-96 \%$. Mean follow-up was $4.7 \pm 1.5$ years. Seven of the 9 studies $[15,18-23]$ referred to the ATA risk classification and this permitted to define separate risk categories. One study [16] referred to AJCC/TNM staging system without mentioning the ATA risk classification. However, this investigation only included patients with small tumor size, extra-thyroidal extension, and no neck lymph node metastasis who were considered at intermediate risk in the separate analysis. In another study [17] only referring to the AJCC/TNM staging system, patients with pT3pT4 tumors or metastasis were defined as high-risk. Finally, although one investigation [22] referred to ATA risk categories, SA rates were only available when considering both intermediate and high-risk patients together and this study was only considered for the overall analysis. 
Table 1

Demographic data and clinical characteristics of study population

\begin{tabular}{|c|c|c|c|c|c|c|c|c|c|}
\hline & $\begin{array}{l}\text { Patients } \\
\text { included } \\
\text { in the } \\
\text { meta- } \\
\text { analysis } \\
(n)\end{array}$ & $\begin{array}{l}\text { Age } \\
\text { (years) }\end{array}$ & $\begin{array}{l}\text { Women } \\
(\%)\end{array}$ & $\begin{array}{l}\text { Extent } \\
\text { of } \\
\text { surgery }\end{array}$ & $\begin{array}{l}\text { RAl } \\
\text { dose } \\
\text { (mCi) }\end{array}$ & $\begin{array}{l}\text { Follow- } \\
\text { up } \\
\text { (years) }\end{array}$ & $\begin{array}{l}\text { Risk } \\
\text { classification }\end{array}$ & $\begin{array}{l}\text { Intermediate } \\
\text { risk }(n)\end{array}$ & $\begin{array}{l}\text { High } \\
\text { risk } \\
(n)\end{array}$ \\
\hline $\begin{array}{l}\text { Caminha } \\
2013 \\
{[15]}\end{array}$ & 152 & $\begin{array}{l}43 \pm \\
14\end{array}$ & 84 & TT & 98 & $\begin{array}{l}13.7 \pm \\
4.1\end{array}$ & ATA & 120 & 32 \\
\hline $\begin{array}{l}\text { Han } \\
2014 \\
{[16]}\end{array}$ & 176 & $50 \pm 9$ & 96 & TT & 83 & 1 & AJCC/TNM & 176 & - \\
\hline $\begin{array}{l}\text { Verburg } \\
2014 \\
{[17]}\end{array}$ & 600 & 45 & 69 & TT & 138 & 10 & AJCC/TNM & - & 600 \\
\hline $\begin{array}{l}\text { Jeon } \\
2014 \\
{[18]}\end{array}$ & 627 & 46 & 87 & TT & 151 & 8 & ATA & 578 & 49 \\
\hline $\begin{array}{l}\text { Rosario } \\
2015 \\
{[19]}\end{array}$ & 180 & 48 & 80 & TT & $\begin{array}{l}30- \\
150\end{array}$ & 1 & ATA & 180 & - \\
\hline $\begin{array}{l}\text { Jeong } \\
2015 \\
{[20]}\end{array}$ & 204 & $\begin{array}{l}44 \pm \\
12\end{array}$ & 92 & TT & 79 & 2 & ATA & 204 & - \\
\hline $\begin{array}{l}\text { Llamas- } \\
\text { Olier } \\
2018 \\
{[21]}\end{array}$ & 389 & $\begin{array}{l}51 \pm \\
11\end{array}$ & 93 & TT & 108 & 1 & ATA & 389 & - \\
\hline $\begin{array}{l}\text { Avram } \\
2019 \\
{[22]}\end{array}$ & 350 & $\begin{array}{l}46 \pm \\
16\end{array}$ & 66 & TT & 105 & $\begin{array}{l}3.3 \pm \\
1.9\end{array}$ & ATA & 350 & \\
\hline $\begin{array}{l}\text { Kim } \\
2019 \\
{[23]}\end{array}$ & 425 & $\begin{array}{l}49 \pm \\
12\end{array}$ & 76 & TT & 108 & 2.3 & ATA & 292 & 133 \\
\hline
\end{tabular}

\section{Assessment of the methodological quality of the included studies}

Figure 2 summarizes the quality assessment of the 9 included studies using Critical Appraisal tools for use in JBI Systematic Reviews. The risk of bias was considered low overall. The domain that showed an unclear risk of bias was "study subjects and setting". This result could be due to slightly different descriptions of patient characteristics among studies.

\section{Successful ablation rate in the overall population}

The SA rate reported in the 9 studies ranged from $51-94 \%$ (Fig. 3), the pooled SA rate was $71 \%$ (95\% confidence interval, $\mathrm{Cl}, 59-83)$ and the heterogeneity was $98.58 \%(P<0.001)$. The funnel plot indicates no publication bias $(P=$ 
0.16) among these studies (see Supplementary material, Figure S1). At meta-regression analysis, there was a negative association between SA rate and mean RAI activity administered (coefficient $=-0.002$; standard error $=0.27$; $\mathrm{t}=3.51 ; P<0.05)$. As shown in Fig. 4, higher RAl activities administered for more clinical severe disease was associated with a lower SA rate. With a mean RAl activity of $83.7 \mathrm{mCi}$, the SA rate was $93.7 \%$, while with a mean RAI activity of $151 \mathrm{mCi}$ the SA rate was $57.5 \%$.

\section{Successful ablation rate in intermediate risk patients}

The SA rate in intermediate risk patients was reported in 7 studies [16,17,19,20-22,24], including 1939 patients. It ranged from $51-94 \%$, the pooled SA rate was $72 \%(95 \% \mathrm{Cl} 59-86)$ and the heterogeneity was $98.4 \%(P<0.001)($ see Supplementary material, Figure S2). The funnel plot indicates no publication bias $(P=0.14)$ among these studies (see Supplementary material, Figure S3). At meta-regression analysis did not find any association between SA and the variables analyzed. Of note, the 350 patients at intermediate and high-risk evaluated by Avram et al. [22] were not included in any subgroup analysis because data could not be evaluated in separate categories but only as a single group.

\section{Successful ablation rate in high-risk patients}

The SA rate according to separate high-risk patients' category has been reported in only 4 studies $[15,17,18,23]$ including 814 patients and ranged from $18-78 \%$. The pooled SA rate was $52 \%(95 \% \mathrm{Cl} 31-74)$ and the heterogeneity was $96.5 \%(P<0.001)$ (see Supplementary material, Figure 44$)$. The funnel plot indicates no publication bias $(P=$ 0.08) among these studies (see Supplementary material, Figure S5). At meta-regression analysis, a negative association between lower SA rate and higher mean RAI activity administered was found (coefficient $=-0.001$; standard error $=0.47 ; \mathrm{t}=4.02 ; P<0.05$ ) (see Supplementary material, Figure S6). For example, with a mean RAI activity of $105 \mathrm{mCi}$ the SA rate was $84 \%$, while with a mean RAl activity of $138 \mathrm{mCi}$ the SA rate was $53 \%$.

\section{Recurrent disease rate at in patients who achieved successful ablation}

A late follow-up (mean $6.4 \pm 1.4$ years) was available in 4 studies $[16,19,20,21]$ including 656 intermediate-risk patients (Table 2). In one study [21] recurrence was defined as new evidence of biochemical or structural disease after any disease-free period, in the other 3 studies $[16,19,20]$ recurrence was cytologically or histologically proven. Recurrence rate after SA ranged from $0-7 \%$ and the pooled recurrent disease rate was $2 \%(95 \% \mathrm{Cl} 0-5)$ (Fig. 5). The heterogeneity was $78.5 \%(P<0.001)$ and the funnel plot indicated no publication bias $(P=0.08)$ among these studies (see Supplementary material, Figure S7). At meta-regression analysis no association between recurrent disease rate and all variables analyzed was found. In contrast, recurrences were observed in 18/121 intermediate risk patients who did not achieve a SA at the first control, with a pooled rate of $14 \%$. 
Table 2

Recurrence at late follow-up in patients with and without successful ablation at early follow-up

\begin{tabular}{|llll|}
\hline & $\begin{array}{l}\text { Patients with recurrence/patients with } \\
\text { successful ablation }\end{array}$ & $\begin{array}{l}\text { Patients with recurrence/patients } \\
\text { without successful ablation }\end{array}$ & $\begin{array}{c}\text { Mean } \\
\text { follow-up } \\
\text { (years) }\end{array}$ \\
\hline Han 2014 [16] & $0 / 165$ & $3 / 11$ & 7.2 \\
\hline $\begin{array}{l}\text { Rosario } 2015 \\
{[19]}\end{array}$ & $4 / 170$ & $1 / 10$ & 5 \\
\hline $\begin{array}{l}\text { Jeong } 2015 \\
{[20]}\end{array}$ & $0 / 104$ & $14 / 100$ & 10 \\
\hline $\begin{array}{l}\text { Llamas-Olier } \\
2018[21]\end{array}$ & $16 / 217$ & NA/172 & 3.5 \\
\hline Values are expressed as number of subjects & & \\
\hline
\end{tabular}

\section{Discussion}

The present investigation refers to 9 studies accounting for 3103 patients at intermediate or high-risk of recurrence. The main finding of our meta-analysis is a $71 \%$ pooled SA rate after RAl treatment and this was indeed higher in intermediate $(72 \%)$ than in high (52\%) risk patients. This is well below the reported SA rates achieved in low-risk patients [24,25], even with the use of relatively low activities $(30 \mathrm{mCi})$ following rhTSH $[25,26]$.

Higher administered activities in high-risk patients were associated with a lower SA rates, suggesting that more extensive disease observed in these high-risk patients are also less sensitive to RAl treatment. This might be related to an impairment in iodine metabolism as documented in patients with a BRAFV600E and TERT mutations [28].

Despite the rigorous inclusion standards, a significant heterogeneity among the evaluated studies was observed. The presence of heterogeneity in a meta-analysis is an expected issue [29]. This result could be explained by different specific end-points considered within each single investigation. It should be also taken into account that, as shown in Table 1, the overall population assessed in every single study referred to a more extensive patient group with heterogeneous clinical characteristics, while the evaluated population included in our analysis matched strict criteria suggesting that the investigations included in the current meta-analysis suffer from different degrees of bias across studies. Nevertheless, the meta-analysis method remains a powerful option to interpret multiple data coming from literature.

A late follow-up was available in 4 studies $[16,19,20,21]$ only considering intermediate risk patients. In patients achieving SA at early follow-up there was a pooled recurrence rate of $2 \%$. In contrast, the pooled recurrence rate was $14 \%$ in patients who did not achieve a SA at early follow-up. These results are in close agreement with the prognostic value of the ATA classification, with the majority of recurrences occurring in patients with some abnormal findings at the first control [27]. Yet, data on high-risk patients were not available to perform a similar analysis in this category.

\section{Conclusion}

The present meta-analysis demonstrates that overall SA rate after RAI treatment in patients at intermediate-high risk of recurrence was $71 \%$ and it was higher in intermediate (72\%) than in high (52\%) risk patients. Further, pooled recurrence rate in patients achieving SA was only $2 \%$ further highlighting the very low probability of recurrence of 
disease once a SA has been obtained after RAI therapy. These data indeed underline the importance of using the concept of ongoing risk assessment that allows to modify the individual prognosis and follow-up strategy according to the results of each control.

\section{Declarations}

\section{Compliance with ethical standards}

\section{Conflict of interest}

The authors declare that they have no conflict of interest.

\section{Ethical approval}

This article does not contain any study with human participants or animals performed by any of the authors.

\section{References}

1. Haugen BR, Alexander EK, Bible KC, Doherty GM, Mandel SJ, Nikiforov YE, et al. 2015 American Thyroid Association Management Guidelines for Adult Patients with Thyroid Nodules and Differentiated Thyroid Cancer: The American Thyroid Association Guidelines Task Force on Thyroid Nodules and Differentiated Thyroid Cancer. Thyroid. 2016;26:1-133.

2. Durante C, Haddy N, Baudin E, Leboulleux S, Hartl D, Travagli JP, et al. Long-term outcome of 444 patients with distant metastases from papillary and follicular thyroid carcinoma: benefits and limits of radioiodine therapy. $J$ Clin Endocrinol Metab. 2006;91:2892-9.

3. Orosco RK, Hussain T, Noel JE, Chang DC, Dosiou C, Mittra E, et al. Radioactive iodine in differentiated thyroid cancer: a national database perspective. Endocr Relat Cancer. 2019;26:795-802.

4. Klain M, Ricard M, Leboulleux S, Baudin E, Schlumberger M. Radioiodine therapy for papillary and follicular thyroid carcinoma. Eur J Nucl Med Mol Imaging. 2002;29 Suppl 2:S479-85.

5. Nascimento C, Borget I, Troalen F, Al Ghuzlan A, Deandreis D, Hartl D, et al. Ultrasensitive serum thyroglobulin measurement is useful for the follow-up of patients treated with total thyroidectomy without radioactive iodine ablation. Eur J Endocrinol. 2013;169:689-93.

6. Lamartina L, Durante C, Filetti S, Cooper DS. Low-risk differentiated thyroid cancer and radioiodine remnant ablation: a systematic review of the literature. J Clin Endocrinol Metab. 2015;100:1748-61.

7. Moher D, Liberati A, Tetzlaff J, Altman DG, PRISMA Group. Preferred reporting items for systematic reviews and meta-analyses: the PRISMA statement. PLoS Med. 2009;(6)7:e1000097.

8. Tuttle M, Morris LF, Haugen B, Shah J, Sosa JA, Rohren E, et al. 2017 Thyroid differentiated and anaplastic carcinoma. In: Amin MB, Edge SB, Greene F, Byrd D, Brookland RK, Washington MK, Gershenwald JE, Compton CC, Hess KR, Sullivan DC, Jessup JM, Brierley J, Gaspar LE, Schilsky RL, Balch CM, Winchester DP, Asare EA, Madera M, Gress DM, Meyer LR, (eds) AJCC Cancer Staging Manual. Eighth edition. Springer International Publishing, New York, New York.

9. Munn Z, Moola S, Riitano D, Lisy K. The development of a critical appraisal tool for use in systematic reviews addressing questions of prevalence. Int J Health Policy Manag. 2014;3:123-8.

10. Nyaga VN, Arbyn M, Aerts M. Metaprop: a Stata command to perform meta-analysis of binomial data. Arch Public Health. 2014;72:39. 
11. Higgins JP, Thompson SG. Quantifying heterogeneity in a meta-analysis. Stat Med. 2002;21:1539-58.

12. Higgins JPT, Green S. Cochrane Handbook for Systematic Reviews of Interventions.Chichester: Wiley; 2008.

13. Ades AE, Lu G, Higgins JPT. Medical Decision Making The Interpretation of Random-Effects Meta-Analysis in Decision Models. Med Decis Making. 2005;25:646.

14. Egger M, Davey Smith G, Schneider M, Minder C. Bias in meta-analysis detected by a simple, graphical test. BMJ. 1997;315:629- 34.

15. Caminha LS, Momesso DP, Vaisman F, Corbo R, Vaisman M. Long-term follow-up of patients with differentiated thyroid cancer who had negative 131 I whole-body scan at first evaluation after treatment. Clin Nucl Med. 2013;38:765-9.

16. Han JM, Kim WG, Kim TY, Jeon MJ, Ryu JS, Song DE, Hong SJ, Shong YK, Kim WB. Effects of low-dose and highdose postoperative radioiodine therapy on the clinical outcome in patients with small differentiated thyroid cancer having microscopic extrathyroidal extension. Thyroid. 2014;24:820-5.

17. Verburg FA, Mäder U, Reiners $C$, Hänscheid H. Long-term survival in differentiated thyroid cancer is worse after low-activity initial post-surgical 131I therapy in both high- and low-risk patients. J Clin Endocrinol Metab. 2014;99:4487-96.

18. Jeon MJ, Kim WG, Park WR, Han JM, Kim TY, Song DE, et al. Modified dynamic risk stratification for predicting recurrence using the response to initial therapy in patients with differentiated thyroid carcinoma. Eur $\mathrm{J}$ Endocrinol. 2013;170:23-30.

19. Rosario PW, Furtado Mde S, Mourão GF, Calsolari MR. Patients with papillary thyroid carcinoma at intermediate risk of recurrence according to American Thyroid Association criteria can be reclassified as low risk when the postoperative thyroglobulin is low. Thyroid. 2015;25:1243-8.

20. Jeong JH, Kong EJ, Jeong SY, Lee SW, Cho IH, Ah Chun K, et al. Clinical outcomes of low-dose and high-dose postoperative radioiodine therapy in patients with intermediate-risk differentiated thyroid cancer. Nucl Med Commun. 2017;38:228-33.

21. Llamas-Olier AE, Cuéllar DI, Buitrago G. Intermediate-risk papillary thyroid cancer: risk factors for early recurrence in patients with excellent response to initial therapy. Thyroid. 2018;28:1311-7.

22. Avram AM, Rosculet N, Esfandiari NH, Gauger PG, Miller BS, Cohen M, et al. Differentiated thyroid cancer outcomes after surgery and activity-adjusted 131I theragnostics. Clin Nucl Med. 2019;44:11-20.

23. Kim HK, Yoon JH, Cho JS, Kwon SY, Yoo SW, Kang HC. The clinical meaning of pre- and post-ablation thyroglobulin levels at first radioiodine therapy in patients with papillary thyroid cancer. Korean $\mathrm{J}$ Intern Med. 2020;35:1164-72.

24. Schlumberger M, Catargi B, Borget I, Deandreis D, Zerdoud S, Bridji B, et al.; Tumeurs de la Thyroïde Refractaires Network for the Essai Stimulation Ablation Equivalence Trial. Strategies of radioiodine ablation in patients with low-risk thyroid cancer. N Engl J Med. 2012;366:1663-73.

25. Alexander EK, Larsen PR. Radioiodine for thyroid cancer--is less more? N Engl J Med. 2012;366:1732-3.

26. Mallick U, Harmer C, Yap B, Wadsley J, Clarke S, Moss L, et al. Ablation with low-dose radioiodine and thyrotropin alfa in thyroid cancer. N Engl J Med. 2012;366:1674-85.

27. Leboulleux S, Rubino C, Baudin E, Caillou B, Hartl DM, Bidart JM, et al. Prognostic factors for persistent or recurrent disease of papillary thyroid carcinoma with neck lymph node metastases and/or tumor extension beyond the thyroid capsule at initial diagnosis. J Clin Endocrinol Metab. 2005;90:5723-9.

28. Liu J, Liu R, Shen X, Zhu G, Li B, Xing M. The genetic duet of BRAF V600E and TERT promoter mutations robustly predicts loss of radioiodine avidity in recurrent papillary thyroid cancer. J Nucl Med. 2020;61:177-82.

Page $11 / 16$ 


\section{Figures}
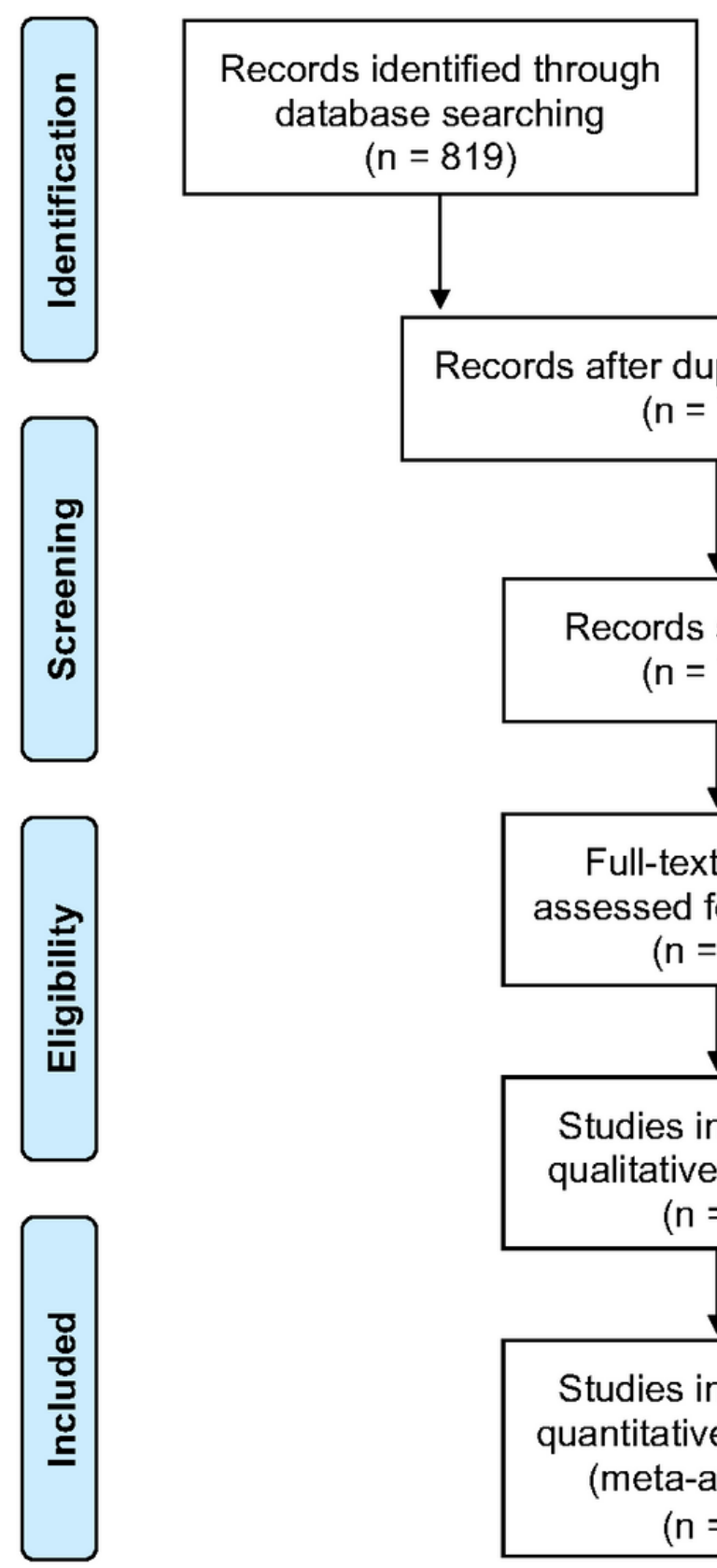

Additional records identified through other sources

$(n=819)$
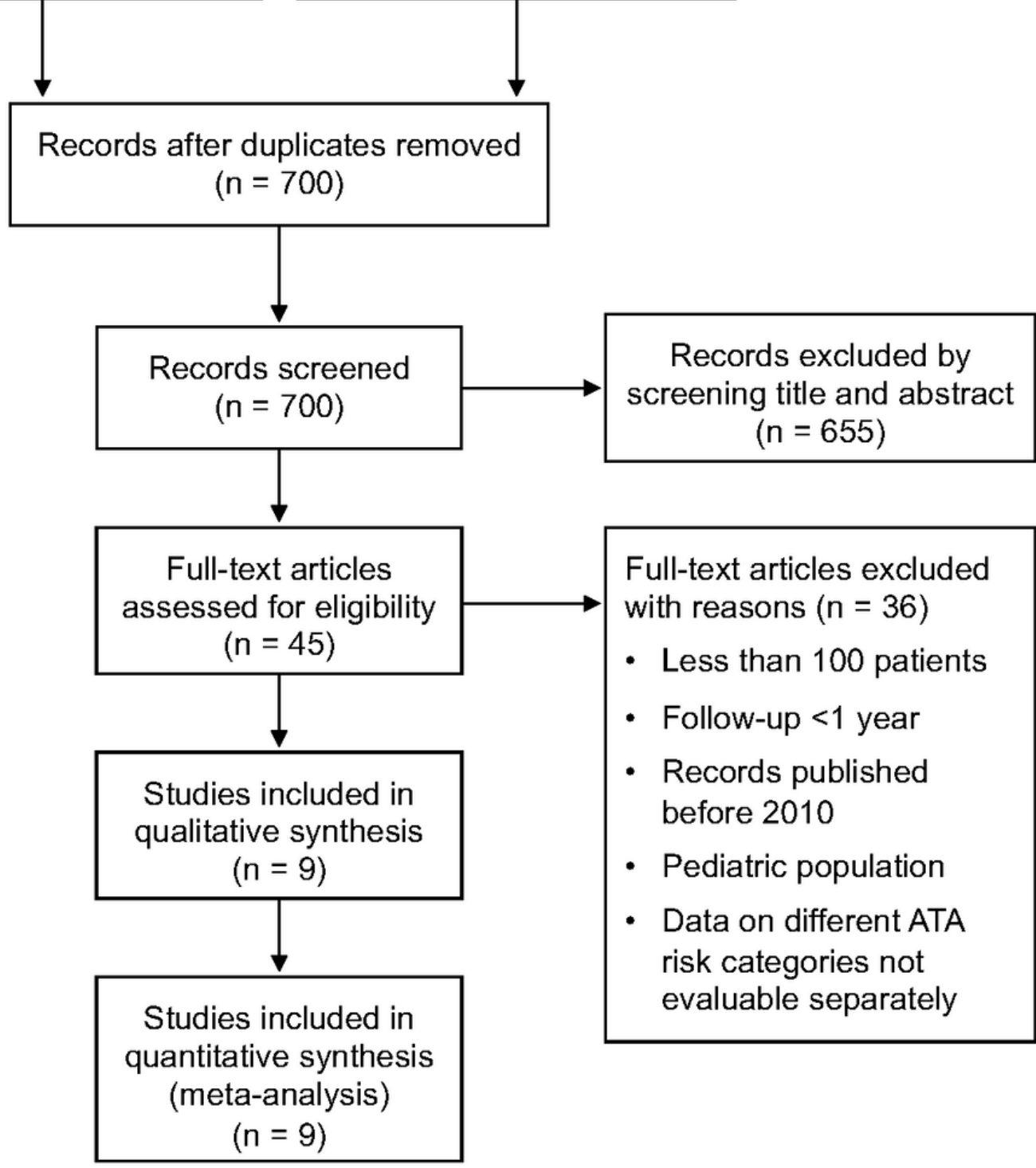

Full-text articles excluded with reasons $(n=36)$

- Less than 100 patients

- Follow-up $<1$ year

- Records published before 2010

- Pediatric population

- Data on different ATA risk categories not evaluable separately

Figure 1

PRISMA flowchart illustrating the study selection process. 


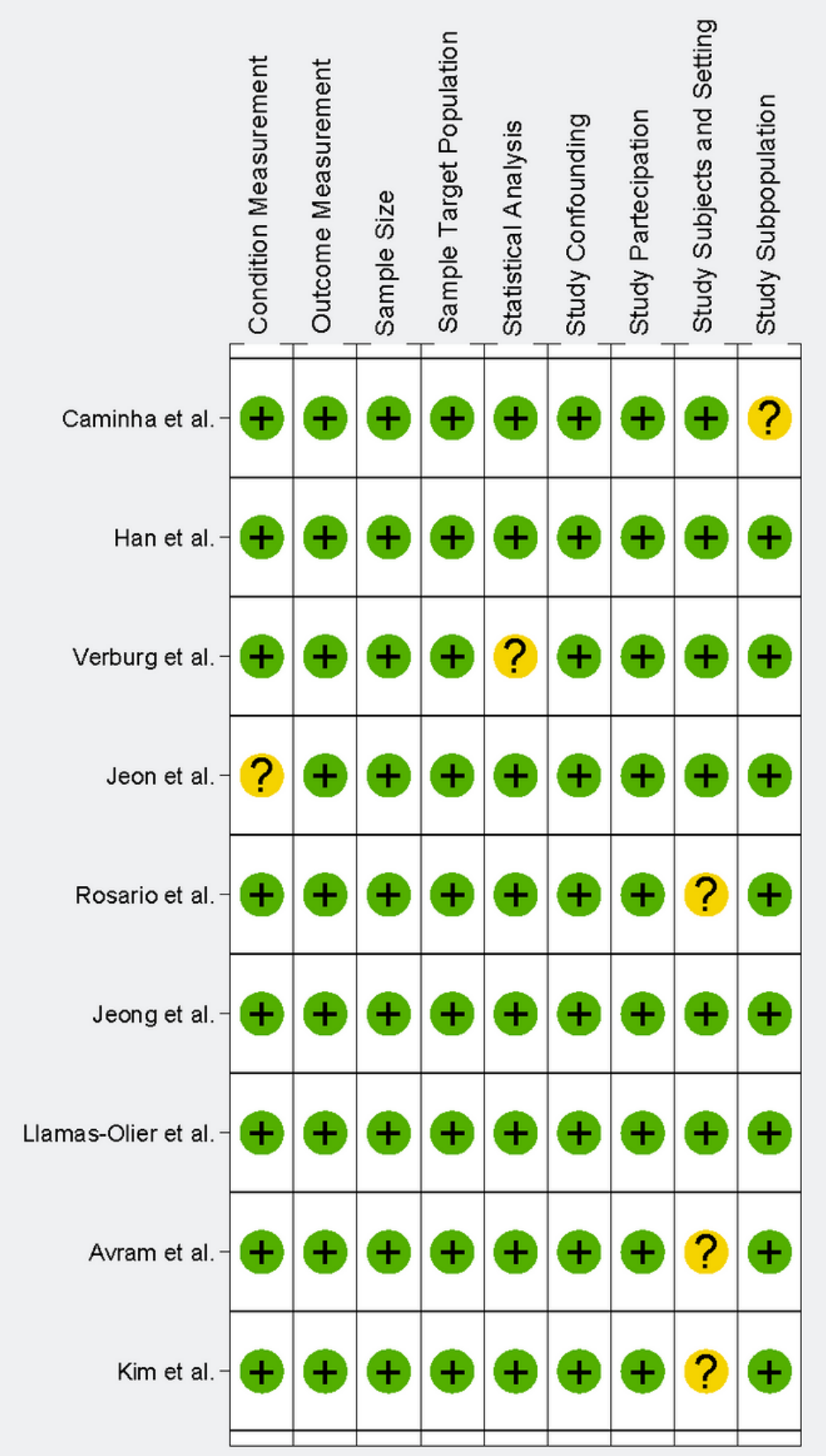

\section{Figure 2}

Methodological quality of the included studies assessed with JBI tool for risk of bias and applicability concerns. The green circle represents low risk of bias, the yellow circle unclear risk of bias and the red circle high risk of bias. 


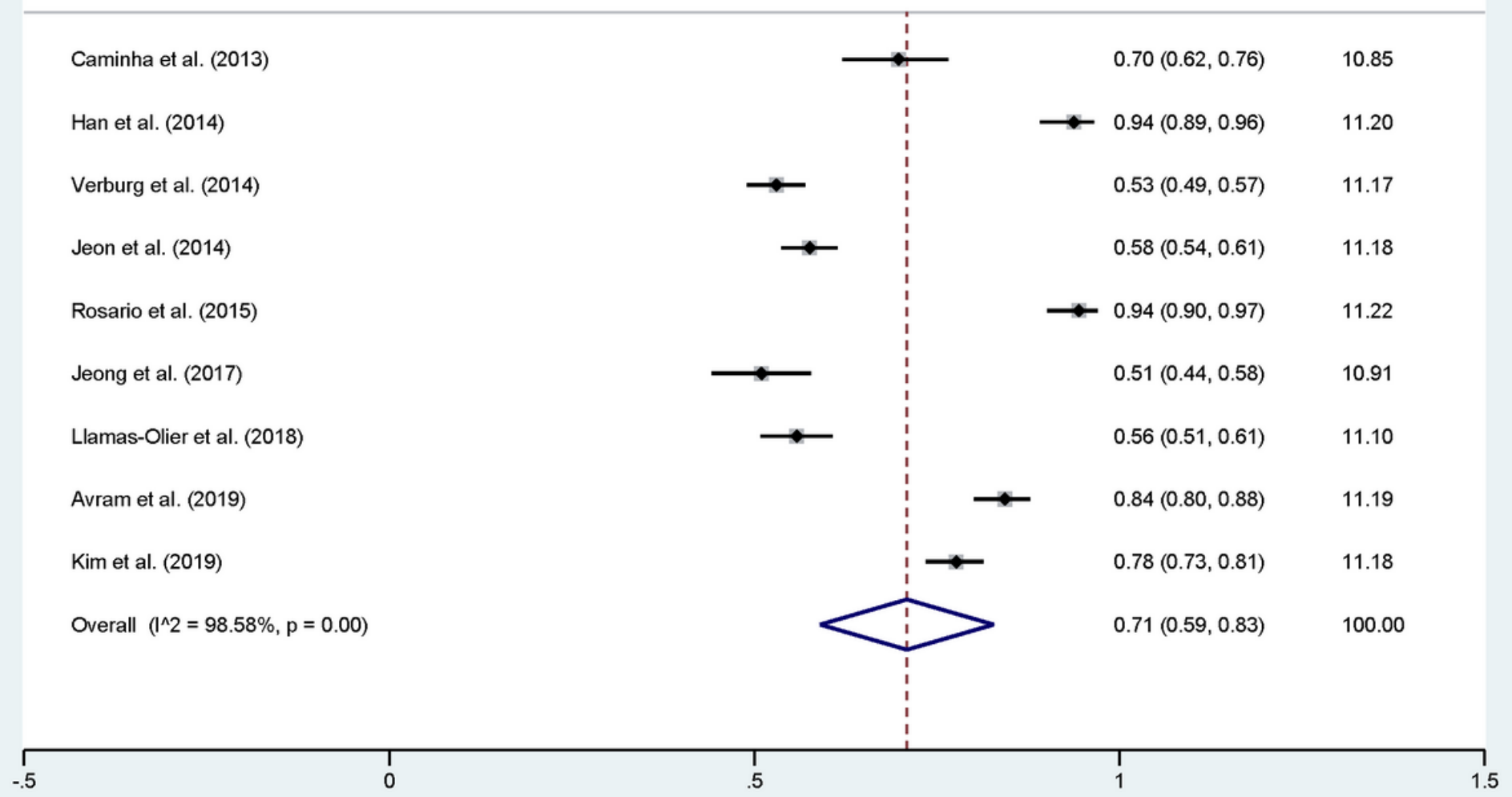

\section{Figure 3}

Forest plot for the successful ablation rate after RAl therapy. Horizontal lines represent $95 \%$ confidence interval of the point estimates. The diamond represents the pooled estimate (size of the diamond $=95 \%$ confidence interval). The solid vertical line represents the reference of no increased risk and the dashed vertical line represents the overall point estimate. 


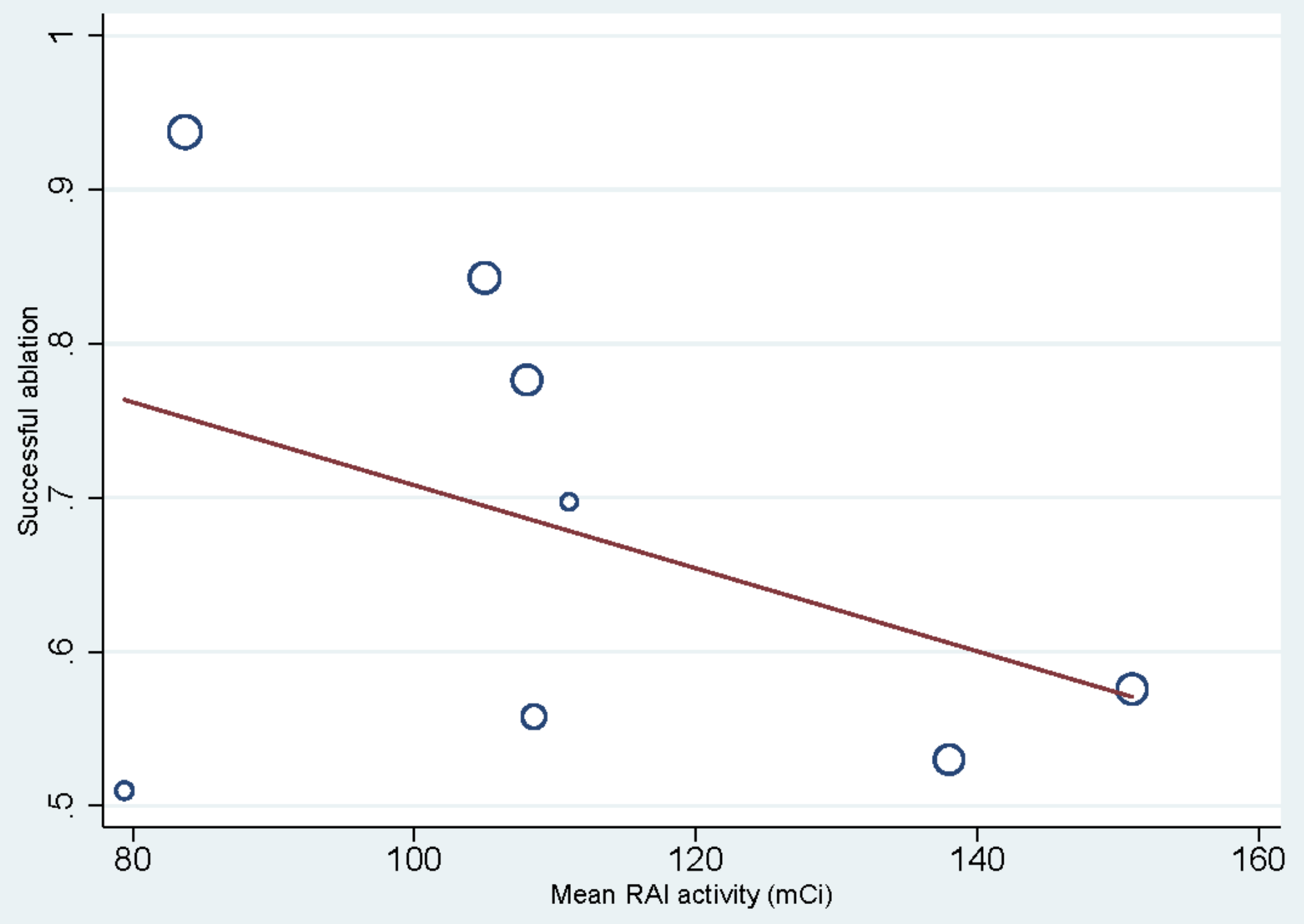

Figure 4

Meta-regression analysis between SA and the mean RAl activity administered. Bubble size for each study is proportional to the inverse of the variance. 


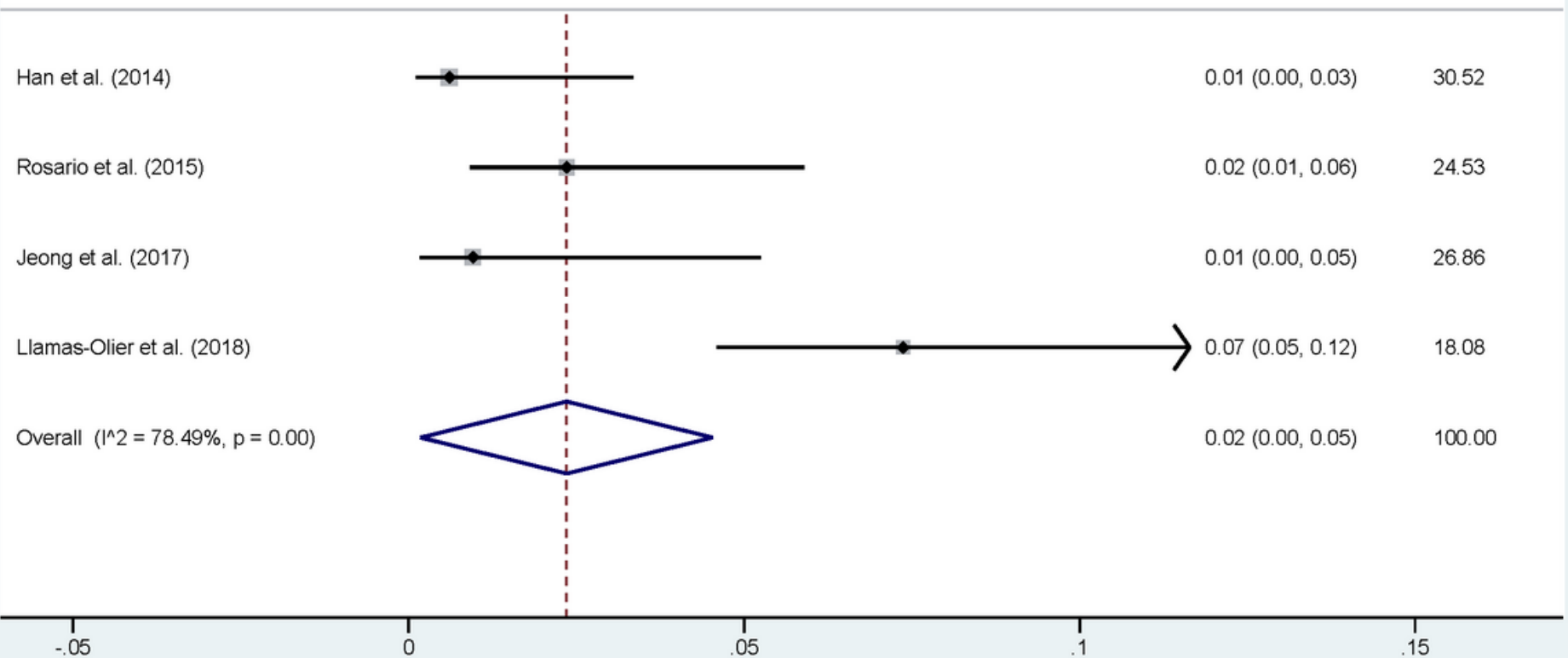

\section{Figure 5}

Forest plot for the persistent/recurrent disease rate at late follow-up in patients who achieved SA. Horizontal lines represent 95\% confidence interval of the point estimates. The diamond represents the pooled estimate (size of the diamond $=95 \%$ confidence interval). The solid vertical line represents the reference of no increased risk and the dashed vertical line represents the overall point estimate.

\section{Supplementary Files}

This is a list of supplementary files associated with this preprint. Click to download.

- FigureS1.pdf

- Figures2.pdf

- Figures3.pdf

- FigureS4.pdf

- Figures5.pdf

- Figures6.pdf

- FigureS7.pdf

- PRISMAChecklist.doc

- Supplementaryfigurelegends.docx 